\title{
Overlarge Gauge Factor Yields a Large Measuring Error for Resistive-Type Stretchable Strain Sensors
}

\author{
Shuang Li, Guodong Liu, Liwu Wang, Guanhui Fang, and Yewang Su*
}

Stretchable strain sensors have recently attracted great attention due to their extensive applications in advanced devices. Plenty of work has been devoted to enlarging the gauge factor (GF) (reaching as high as $10^{7}$ ), since the conventional wisdom holds that a high GF indicates a low limit of detection and a high resolution of a sensor. Here, the effects of the GF on the measurement accuracy is investigated for resistive-type strain sensors, via the basic approach based on Ohm's law and the commonly used method of Wheatstone bridge, respectively. It is found that a bigger GF is not always better for improvement of the measurement accuracy, which is contrary to conventional wisdom. Overlarge gauge factor yields a large measuring error for resistive-type stretchable strain sensors (reaching the measuring error of $50 \%$ for GF $=5 \times 10^{4}$ under a typical measurement condition). This finding is of much significance for providing theoretical guidance for the sensor design and avoid more efforts on overlarge GF.
In recent years, flexible strain sensors with large sensing range (also known as stretchable strain sensors) have attracted great attentions due to their extensive applications in wearable electronics, ${ }^{[1]}$ e-skin, ${ }^{[2]}$ soft robotics, ${ }^{[3]}$ healthcare devices, ${ }^{[4]}$ and other advanced devices. Based on "Web of Science," more than 5000 papers related to flexible strain sensors have been published in the past decade. Thousands of new-type flexible strain sensors based on different sensing mechanisms have emerged, such as resistive effect, ${ }^{[1,3,5]}$ capacitive effect, ${ }^{[6]}$ piezoelectric effect, ${ }^{[7]}$ and

Dr. S. Li, Dr. G. Liu, Prof. Y. Su

State Key Laboratory of Nonlinear Mechanics

Institute of Mechanics

Chinese Academy of Sciences

Beijing 100190, China

E-mail: yewangsu@imech.ac.cn

Dr. S. Li, Dr. G. Liu, Prof. Y. Su

School of Engineering Science

University of Chinese Academy of Sciences

Beijing 100049, China

Prof. L. Wang, Prof. G. Fang

Beijing Institute of Space Mechanics \& Electricity

Beijing 100094, China

Prof. Y. Su

State Key Laboratory of Structural Analysis for Industrial Equipment

Department of Engineering Mechanics

Dalian University of Technology

Dalian 116024, China

The ORCID identification number(s) for the author(s) of this article can be found under https://doi.org/10.1002/aelm.202000618.

DOI: 10.1002/aelm.202000618 triboelectric effect. ${ }^{[8]}$ Among these sensors, the resistive-type strain sensor is the most popular one, because of the simplicity of its structure design and its strong anti-jamming capability. ${ }^{[9,10]}$ Gauge factor (GF) is regarded as the major indicator to measure the performance of strain sensors. The conventional wisdom holds that a high GF indicates a low limit of detection and a high resolution of a sensor. ${ }^{[11]}$ Therefore, much work has been devoted to enlarging the GF of sensors. For example, conventional metallic strain gauge for the small strain sensing has a small GF (usually only about 2.0), ${ }^{[1,12]}$ and researchers have developed semiconductor strain gauges with higher GF values (more than 100) since $1957,{ }^{[13,14]}$ which can significantly increase the magnitude of the output signal and improve the measurement accuracy. In the investigation of stretchable strain sensors, which is a hot topic recently, the record of the GF has been updated rapidly to as high as $10^{7}[1,3,5,15-49]$ (Figure 1) with the continuing efforts of the scientists. However, is the conclusion, that the GF is the bigger the better, correct for the ultrastretchable strain sensors?

In this study, the effects of the GF on the measurement accuracy is studied via theoretical models for resistive-type strain sensors. The basic approach of direct measuring the resistance and its change based on Ohm's law and the commonly used method of Wheatstone bridge are adopted for the analysis, respectively. It is found that the overlarge GF yields a large measuring error (reaching $50 \%$ for $\mathrm{GF}=5 \times 10^{4}$ under a typical measurement condition), which is contrary to the conventional wisdom of researchers. This finding is of much importance because that a lot of research has been focusing on enlarging the GF of strain sensors. ${ }^{[15,20,28,32,35-37,40-47,49]}$ This conclusion can provide theoretical guidance for the sensor design and avoid more efforts on overlarge GF.

First of all, the unconventional finding can be understood by an intuitive logic. For overlarge GF and large strain, the resulted resistance of strain sensors should be very large. The measured current/voltage, in this case, must be very small, and may approach to their detection limit, leading to a large measuring error of strain. The quantitative and strict analysis are provided in the following parts. The resistance of resistivetype strain sensors changes as a response to the mechanically applied strain. Let $R_{0}$ be the initial resistance of a resistive-type strain sensor. It becomes $R$ when the sensor is subjected to the applied strain $\varepsilon$. The GF $K$ is defined as the ratio of the change of the relative resistance to the applied strain, ${ }^{[22]}$ i.e. 


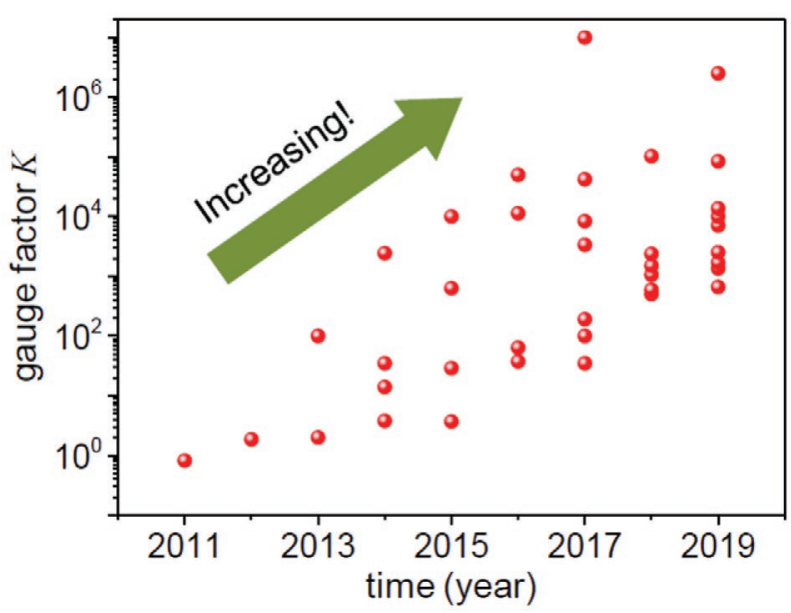

Figure 1. The rapid growth of the GF of stretchable strain sensors in recent years.

$\frac{R-R_{0}}{R_{0}}=K \varepsilon$

Here, the GF $K$ can be a constant or a function of applied strain ( $K>0$, according to the existing researches for resistivetype stretchable strain sensors), while the function form does not affect all the following derivations. The basic approach to measure the resistance and its change is based on Ohm's law

$R=\frac{U_{0}}{I}$

Here, $U_{0}$ is a constant applied voltage; $I$ is the current to be measured, as depicted in Figure 2a. The measuring error of the current $\delta I$, which is a constant that decided by the measuring equipment, yields the error of the obtained resistance $\delta R$. The relationship $(R+\delta R)(I+\delta I)=U_{0}$ can be obtained according to Equation (2), which gives

$\delta R=-\frac{R^{2}}{U_{0}} \delta I$

Here, the high-order term is neglected as it is much less than the low-order term. The measuring error of the strain $\delta \varepsilon$ is proportional to $\delta R$, i.e., $\delta \varepsilon=\delta R /\left(R_{0} K\right)$, which, together with Equations (1) and (3), yields

$\left|\frac{\delta \varepsilon}{\varepsilon}\right|=\frac{R_{0}|\delta I|}{U_{0}} \frac{(1+K \varepsilon)^{2}}{K \varepsilon}$

Equation (4) shows that the relative error $|\delta \varepsilon / \varepsilon|$ of the finally obtained strain is a function of the product of the gauge factor and the applied strain, $K \varepsilon$, while $R_{0}|\delta I| / U_{0}$ is the coefficient.

Figure $2 \mathrm{~b}$ studied the influence of the GF $K$ on the relative error $|\delta \varepsilon / \varepsilon|$ quantitatively, with the reasonable parameters $\delta I= \pm 0.1 \mu \mathrm{A}, U_{0}=1 \mathrm{~V}, R_{0}=100 \Omega$, and $\varepsilon=100 \%$. The relative error of the strain is less than $1 \%$, when GF $K$ is in the range of $0.001-1000$. However, when the GF $K$ is out of this range, the error increases rapidly (reaching $50 \%$ at $K=2 \times 10^{-5}$ or $K=5 \times 10^{4}$ ). It can be seen clearly that overlarge GF reduces the measuring accuracy of strain sensors, as well as excessively low GF. Equation (4) shows that the relative error $|\delta \varepsilon / \varepsilon|$ is linearly proportional to the measuring error of the current $\delta I$. The measuring instrument with a high accuracy (small $\delta I$ ) and a high applied voltage $U_{0}$ is obviously beneficial to reduce the relative error $|\delta \varepsilon / \varepsilon|$. However, the measuring instrument with a high accuracy and a high applied voltage is usually bulky and does not fit the requirements of portable electronics. In addition, it is unwise to try to reduce the measuring error by immensely reducing $R_{0}$, because in this way, the contact resistance cannot be ignored and the whole measuring system will become more unstable. On the other hand, the optimization of the GF, which will be discussed in the following text, is independent of the measuring error of the current $\delta I$, the initial resistance $R_{0}$ and the applied voltage $U_{0}$. This finding is much different from the conventional wisdom that the high GF indicates a low limit of detection and a high resolution of a sensor. ${ }^{[11]}$ It can be understood by the following mechanism. The combination of Equations (1) and (2) give the relationship between the dimensionless current $I /\left(U_{0} / R_{0}\right)$ and the product of the gauge factor and the applied strain $K \varepsilon$ as $I /\left(U_{0} / R_{0}\right)=1 /(1$ $+K \varepsilon$ ), which is plotted in Figure 2c. For excessively low GF, the change of the resistance $R-R_{0}$ may be so small that the dimensionless current $I /\left(U_{0} / R_{0}\right)$ approaches the constant 1 . The corresponding change of the current approaches or becomes smaller than the order of the measuring error of the current $\delta I$. For the overlarge GF, the change of the resistance $R-R_{0}$ and its total value $R$ are very large, even though its original value $R_{0}$
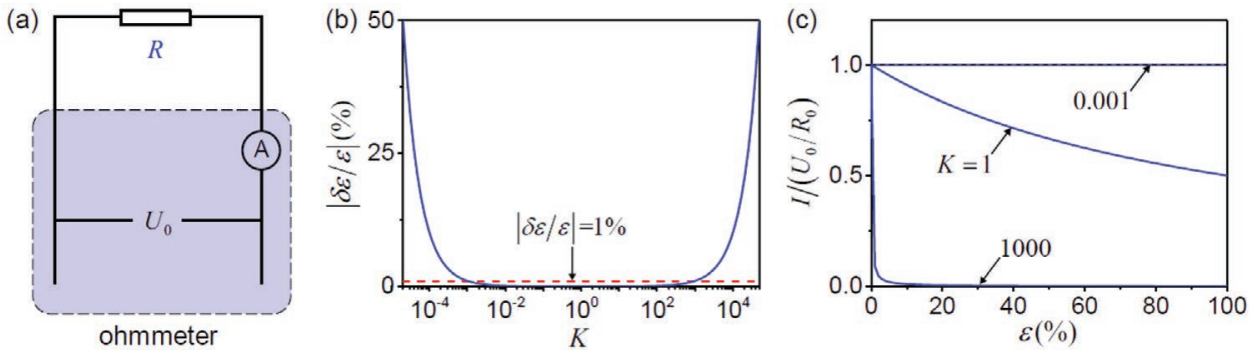

Figure 2. The analysis via the basic approach of direct measuring the resistance and its change based on Ohm's law. a) Schematic diagram of measuring the resistance change of strain sensors based on Ohm's law. b) The influence of the GF $K$ on the relative error $|\delta \varepsilon / \varepsilon|$ with reasonable parameters $\left(\delta I= \pm 0.1 \mu \mathrm{A}, U_{0}=1 \mathrm{~V}, R_{0}=100 \Omega\right.$, and $\left.\varepsilon=100 \%\right)$. c) Curves of the dimensionless current $I /\left(U_{0} / R_{0}\right)$ versus the applied strain $\varepsilon$ with different gauge factors $(\mathrm{GF}=0.001,1$, and 1000). 
is small. The dimensionless current $I /\left(U_{0} / R_{0}\right)$ approaches the constant 0 . The current $I$ and its change approach or become smaller than the magnitude of the measuring error of the current $\delta$ I. Therefore, the measuring accuracy is much reduced for both the two cases. However, many recent literatures published in high-impact journals reported the ultrastretchable strain sensors with the GF being as high as $10^{7}$ and sensing range beyond $100 \%$. Such a high GF was highlighted in the title or the conclusions of the literatures as an advantage. These expressions should be reconsidered and optimized. The derivative of Equation (4)

$\frac{d}{d(K \varepsilon)}\left|\frac{\delta \varepsilon}{\varepsilon}\right|=\frac{R_{0}|\delta I|}{U_{0}}\left[1-\frac{1}{(K \varepsilon)^{2}}\right]$

yields that the optimal case is $K \varepsilon=1$. Therefore, the optimal GF is related to the range of the strain measurement. For the measuring requirement of small applied strain $\varepsilon=0.1 \%$ in many engineering fields, the GF $K$ is the bigger the better in the range [0: 1000], so people should try to improve the GF to get close to the optimal value. For ultrastretchable strain sensors $\varepsilon=100 \%$, the optimal GF $K$ is 1 , rather than large values such as $10^{7}$. Whether there was a large measuring error in previous studies with such high GF is also related to the measuring instruments according to Equation (4). The measuring instrument with a high accuracy, which is bulky and does not fit the requirements of portable electronics, has to be used to get a reliable result for ultrastretchable strain sensors with the large GF. ${ }^{[42,49]}$ However, the accuracy of the existing instrument is limited, and so far the commercial measuring instrument with the highest accuracy is only at the level of pA, which does not work as well for a stretchable strain sensor with a GF of $10^{9}$ or even larger. There will be similar problems for other measuring methods with constant current source. Because of the overlarge $\mathrm{GF}$, the measuring voltage will be very large, and may exceed the measuring range. In order to aviod exceeding the voltage range, the constant current source $I$ must be very small, and may approach to the accuracy $\delta I$, leading to a large measuring error of strain. Therefore, there is still an optimal GF instead of being the bigger the better.

In scientific research and industrial application, Wheatstone bridge is the most commonly used circuit for resistive-type strain sensors to achieve signal amplification and temperature compensation. Without loss of generality, a quarter bridge is taken as an example to illustrate that an overlarge GF also reduces the accuracy of the measurement. Let $R_{1}$ denote the resistance of the strain sensor, $R_{2}, R_{3}$, and $R_{4}$ denote the other three resistances of the bridge, and $U_{0}$ denote the source voltage of the bridge (Figure 3a). The output voltage of the bridge circuit $U_{\mathrm{m}}=\left(R_{1} R_{3}-R_{2} R_{4}\right) /\left[\left(R_{1}+R_{2}\right)\left(R_{3}+R_{4}\right)\right] U_{0}^{[50]}$ changes with the change of the resistance of the strain sensor $R_{1}$. In this way, the Wheatstone bridge converts the signal of resistance into the signal of voltage. For simplicity, all the four resistances usually equal to $R_{0}$ for the initial regime with zero strain. The sensor $R_{1}$ becomes $(1+K \varepsilon) R_{0}$ when it is subjected to the applied strain $\varepsilon$, while others remain unchanged. The output voltage becomes

$$
U_{\mathrm{m}}=\frac{K \varepsilon}{2(2+K \varepsilon)} U_{0}
$$
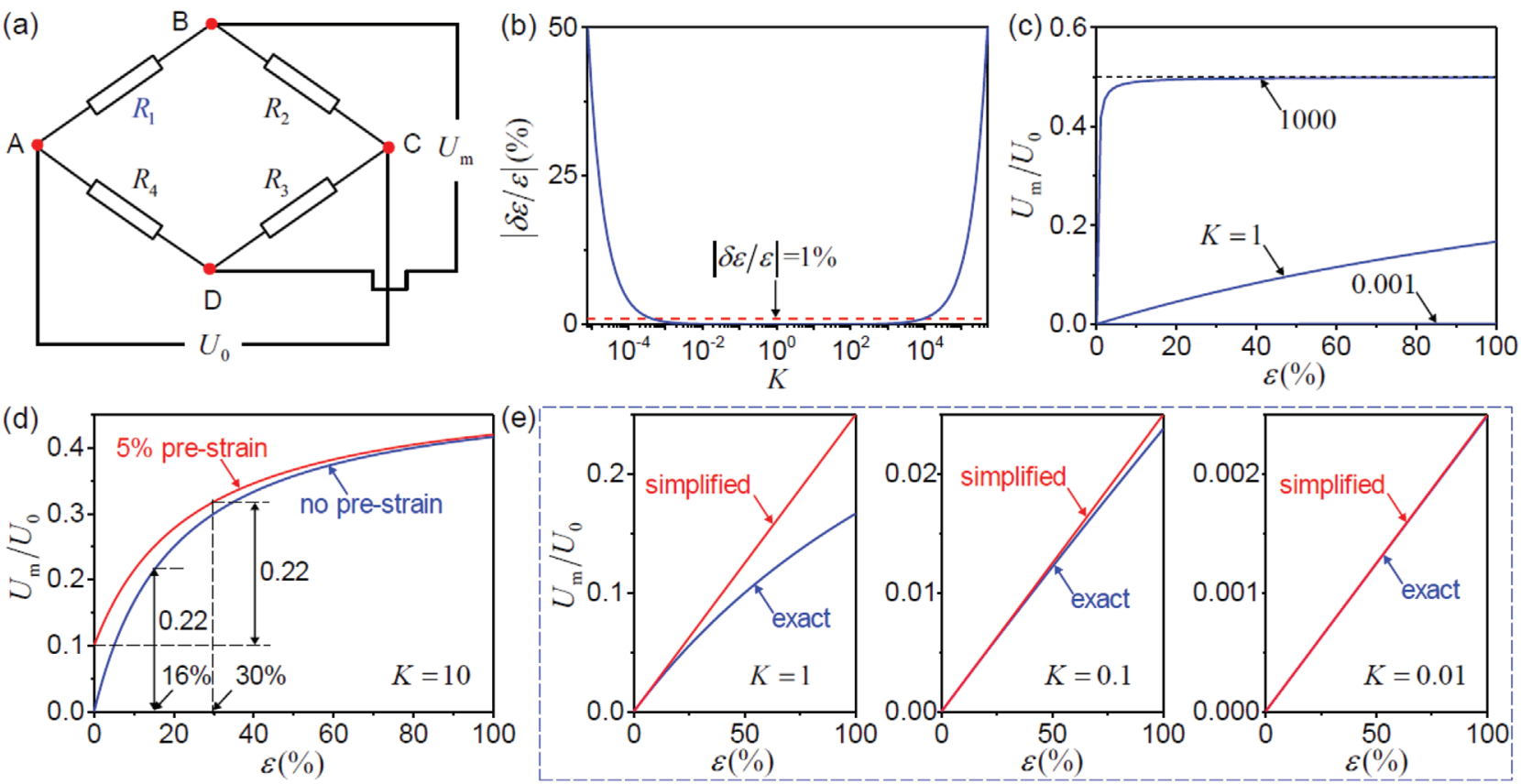

Figure 3. The analysis via the commonly used method of Wheatstone bridge. a) Schematic diagram of measuring the resistance change of strain sensors with Wheatstone bridge. b) The effects of the GF $K$ on the relative error $|\delta \varepsilon / \varepsilon|$ with reasonable parameters $\left(\delta U_{\mathrm{m}}= \pm 1 \mu \mathrm{V}, U_{0}=1 \mathrm{~V}\right.$, and $\left.\varepsilon=100 \%\right)$. c) Curves of the dimensionless output voltage $U_{m} / U_{0}$ versus the applied strain with different gauge factors $(G F=0.001,1$, and 1000$)$. d) Comparison of dimensionless output voltage of a sensor with $5 \%$ prestrain and a sensor without prestrain. e) The effects of the GF $K$ on the linearity of the relation between the dimensionless output voltage $U_{m} / U_{0}$ and the applied strain $\varepsilon$ (from left to right, GF $=1,0.1$, and 0.01 ). 
which gives the expression of the strain as

$\varepsilon=\frac{2}{K}\left(\frac{1}{1-2 U_{\mathrm{m}} / U_{0}}-1\right)$

The measuring error of the strain $\delta \varepsilon$ comes from the measuring error of the voltage $\delta U_{\mathrm{m}}$. The replacement of $U_{\mathrm{m}}$ by $U_{\mathrm{m}}+\delta U_{\mathrm{m}}$ in Equation (7) gives $\varepsilon+\delta \varepsilon$, which, together with Equation (7) yields

$\left|\frac{\delta \varepsilon}{\varepsilon}\right|=\frac{(K \varepsilon+2)^{2}}{K \varepsilon} \frac{\left|\delta U_{\mathrm{m}}\right|}{U_{0}}$

Here, the high-order terms are neglected as it is much less than the low-order term. Equation (8) shows that the relative error $|\delta \varepsilon| \varepsilon \mid$ of the finally obtained strain is also a function of the product of the gauge factor and the applied strain $K \varepsilon$.

Figure $3 \mathrm{~b}$ studied the effects of the GF $K$ on the relative error $|\delta \varepsilon / \varepsilon|$, with the reasonable parameters $\delta U_{\mathrm{m}}= \pm 1 \mu \mathrm{V}, U_{0}=1 \mathrm{~V}$ and $\varepsilon=100 \%$. When the GF $K$ is in the range of $4 \times 10^{-4}-10^{4}$, the relative error of the strain is less than $1 \%$. Nevertheless, the error increases rapidly (reaching $50 \%$ at $K=8 \times 10^{-6}$ or $K=5 \times$ $10^{5}$ ), when GF is out of this range. The conclusion found in the approach of direct measuring the resistance and its change based on Ohm's law holds here that overlarge GF and excessively low GF reduce the measuring accuracy of strain sensors. Equation (8) shows that the relative error $|\delta \varepsilon / \varepsilon|$ is linearly proportional to the measuring error of the voltage $\delta U_{\mathrm{m}}$. The measuring instrument with a high accuracy (small $\delta U_{\mathrm{m}}$ ) and a high applied voltage $U_{0}$ is obviously beneficial to reduce the relative error $\mid \delta \varepsilon / \varepsilon$. However, the measuring instrument with a high accuracy and a high applied voltage is usually bulky and does not fit the requirements of portable electronics. On the other hand, the optimization of the GF, which will be discussed in the following text, is independent of the measuring error of the voltage $\delta U_{\mathrm{m}}$ and the applied voltage $U_{0}$. The mechanism (Figure 3c) is similar to that of the above measuring approach. For excessively low GF, the change of the resistance $R_{1}-R_{0}$ may be so small that the corresponding output voltage $U_{\mathrm{m}}$ approaches zero or becomes smaller than the magnitude of the measuring error of the current $\delta U_{\mathrm{m}}$. For the overlarge $\mathrm{GF}$, the change of the resistance $R_{1}-R_{0}$ and its total value $R_{1}$ become much larger than $R_{0}$, even though the four resistances in the Wheatstone bridge equal to each other in the original regime. The output voltage $U_{\mathrm{m}}$ approaches the constant value of $U_{0} / 2$ rapidly. The change of $U_{\mathrm{m}}$ then may become smaller than the order of the measuring error of the voltage $\delta U_{\mathrm{m}}$. Hence, the measuring accuracy of Wheatstone bridge is also much reduced for both the two cases. The optimal regime can be obtained by the derivative of Equation (8)

$\frac{d}{d(K \varepsilon)}\left|\frac{\delta \varepsilon}{\varepsilon}\right|=\frac{(K \varepsilon)^{2}-4}{(K \varepsilon)^{2}} \frac{\left|\delta U_{\mathrm{m}}\right|}{U_{0}}$

which yields that the optimal case is $K \varepsilon=2$. Therefore, the optimal GF is related to the range of the strain measurement. For small applied strain $\varepsilon=1 \%$, the GF $K$ is the bigger the better in the range [0: 200]. For ultrastretchable strain sensors $\varepsilon=100 \%$, the optimal GF $K$ is 2 , rather than large values such as $10^{7}$. It is worth noting that the optimal GF $K=2$ is specifically for the quater-bridage circuit, while it would be $K=1$ for the half-bridge circuit. The optimal GF is related to the measuring system. However, they are all at the order of $\approx 1$, which allows that an optimal ultrastretchable strain sensor with a GF $K \approx 1$ gives quasioptimal accuracy for various measuring circuits.

Finally, we reveal another disadvantage of the overlarge GF for wearable applications of stretchable strain sensors. Equation (6) shows that the accurate relationship between the output voltage $U_{\mathrm{m}}$ and the applied strain $\varepsilon$ is strongly nonlinear (the blue curve in Figure 3d). The problem caused by the nonlinearity is analyzed in Figure $3 \mathrm{~d}(\mathrm{GF}=10)$. When installing the strain sensors onto the sensing target, such as the body skin or organs, the practical operation usually cannot ensure that the installing regime of the sensors is strain free, which is unlike the traditional industrial measurements. The operation of the zero clearing is used for a sensor with $5 \%$ prestrain at the installing regime (the red curve in Figure 3d). The measuring value of the increment of $U_{\mathrm{m}} / U_{0}$ is 0.22 for $30 \%$ applied strain. However, by the comparison of the measuring value $(0.22)$ and the blue curve, the finally measured strain is obtained as $16 \%$, while the real applied strain is $30 \%$. The large mearing error is completely attributed to the strong nonlinearity of the system. It should be noted that this nonlinear problem does not exist in the measurement based on Ohm's law. If the relationship between the output $U_{\mathrm{m}} / U_{0}$ and the strain is linear, the measured strain will be insensitive to the pre-strain of the sensor.

Therefore, the use of the measuring system composed of Wheatstone bridge and the strain sensor usually requires $K \varepsilon \ll 1$. Equation (6) is then simplified to

$U_{\mathrm{m}} \approx \frac{U_{0}}{4} K \varepsilon$

which gives a linear relationship between the output voltage $U_{\mathrm{m}}$ and the applied strain $\varepsilon$. It is easy for the conventional sensors with the small sensing range and the low GF to meet the requirements $K \varepsilon \ll 1(K \approx 1, \varepsilon \approx 1 \%)$. However, the case is much different for stretchable sensors with the high GF. Figure 3e analyzed the effects of the GF $K$ on the linearity of the relation between the output voltage $U_{\mathrm{m}}$ and the applied strain $\varepsilon$ according to the exact expression Equation (6). The cases for $K \leq 0.1$ are acceptable, while the nonlinearity of the cases for $K>0.1$ is relatively strong. Figure 3e clearly shows that the large GF destroys the linearity the measuring system composed of Wheatstone bridge and the strain sensor, therefore reduces the measuring accuracy. It should be noted that, all these derivations do not involve specific sensing mechanisms and do not introduce any new physical hypothesis, so the model is universal to various resistive-type stretchable strain sensors. The sensing materials include carbon-based materials (carbon nanotubes, grapheme, carbonized silk, and carbon black), metal nanowires, etc.

In conclusion, the effects of the GF on the measurement accuracy is investigated for resistive-type strain sensors, via the basic approach based on Ohm's law and the commonly used method of Wheatstone bridge, respectively. In contrast to conventional wisdom, it is found that the GF is not the bigger the better for the improvement of the measurement accuracy. 
The overlarge gauge factor yields a large measuring error for stretchable strain sensors (reaching the measuring error of $50 \%$ for $\mathrm{GF}=5 \times 10^{4}$ under a typical measurement condition). This finding is of much significance since researchers have spent a lot of energy on enlarging the GF of stretchable strain sensors. This finding can provide theoretical guidance for the sensor design and avoid more efforts on overlarge GF. Besides the GF, sensing range, repeatability, linearity, hysteresis, etc., should be considered simultaneously for practical applications of stretchable strain sensors.

\section{Acknowledgements}

Y.S. gratefully acknowledges the support from the National Natural Science Foundation of China (Grants 11772331 and 11572323), Beijing Municipal Science and Technology Commission (Z191100002019010), Beijing Municipal Natural Science Foundation (No. 2202066), Key Research Program of Frontier Sciences Chinese Academy of Sciences (ZDBS-LY-JSC014), Chinese Academy of Sciences via the "Hundred Talent Program", Strategic Priority Research Program of the Chinese Academy of Sciences (No. XDB22040501), Beijing Institute of Space Mechanics \& Electricity, and the State Key Laboratory of Structural Analysis for Industrial Equipment, Dalian University of Technology (No. GZ19102).

\section{Conflict of Interest}

The authors declare no conflict of interest.

\section{Author Contributions}

S.L. and Y.S. conceived the concept. S.L., G.L., L.W., G.F., and Y.S. conducted the theoretical derivation and discussed all the data. S.L. and Y.S. prepared the manuscript. Y.S. supervised the project.

\section{Keywords}

gauge factor, stretchable strain sensors, wearable electronics

Received: June 15, 2020 Revised: August 8, 2020 Published online:

[1] T. Yamada, Y. Hayamizu, Y. Yamamoto, Y. Yomogida, A. IzadiNajafabadi, D. N. Futaba, K. Hata, Nat. Nanotechnol. 2011, 6, 296.

[2] D. Son, J. Kang, O. Vardoulis, Y. Kim, N. Matsuhisa, J. Y. Oh, J. W. F. To, J. W. Mun, T. Katsumata, Y. X. Liu, A. F. McGuire, M. Krason, F. Molina-Lopez, J. Ham, U. Kraft, Y. Lee, Y. Yun, J. B. H. Tok, Z. N. Bao, Nat. Nanotechnol. 2018, 13, 1057.

[3] Y. Cheng, R. R. Wang, J. Sun, L. Gao, Adv. Mater. 2015, 27, 7365

[4] L. H. Li, H. Y. Xiang, Y. Xiong, H. Zhao, Y. Y. Bai, S. Q. Wang, F. Q. Sun, M. M. Hao, L. Liu, T. Li, Z. H. Peng, J. Q. Xu, T. Zhang, Adv. Sci. 2018, 5, 1800558.

[5] M. Amjadi, A. Pichitpajongkit, S. Lee, S. Ryu, I. Park, ACS Nano 2014, 8, 5154.

[6] Z. F. Liu, S. Fang, F. A. Moura, J. N. Ding, N. Jiang, J. Di, M. Zhang, X. Lepro, D. S. Galvao, C. S. Haines, N. Y. Yuan, S. G. Yin, D. W. Lee,
R. Wang, H. Y. Wang, W. Lv, C. Dong, R. C. Zhang, M. J. Chen, Q. Yin, Y. T. Chong, R. Zhang, X. Wang, M. D. Lima, R. OvalleRobles, D. Qian, H. Lu, R. H. Baughman, Science 2015, 349, 400.

[7] J. Zhou, Y. D. Gu, P. Fei, W. J. Mai, Y. F. Gao, R. S. Yang, G. Bao, Z. L. Wang, Nano Lett. 2008, 8, 3035.

[8] X. D. Wang, Y. F. Zhang, X. J. Zhang, Z. H. Huo, X. Y. Li, M. L. Que, Z. C. Peng, H. Wang, C. F. Pan, Adv. Mater. 2018, 30, 1706738.

[9] T. S. D. Le, J. N. An, Y. Huang, Q. Vo, J. Boonruangkan, T. Tran, S. W. Kim, G. Z. Sun, Y. J. Kim, ACS Nano 2019, 13, 13293.

[10] L. Pan, G. Liu, W. X. Shi, J. Shang, W. R. Leow, Y. Q. Liu, Y. Jiang, S. Z. Li, X. D. Chen, R. W. Li, Nat. Commun. 2018, 9, 3813.

[11] Y. F. Liu, Y. Q. Li, P. Huang, N. Hu, S. Y. Fu, Adv. Electron. Mater. 2018, 4, 6

[12] W. B. Dobie, P. C. G. Isaac, Electric Resistance Strain Gauges, English Universities Press, London 1948.

[13] W. P. Mason, R. N. Thurston, J. Acoust, Soc. Am. 1957, 29, 1096.

[14] A. A. Barlian, W. T. Park, J. R. Mallon, A. J. Rastegar, B. L. Pruitt, Proc. IEEE 2009, 97, 513.

[15] M. Amjadi, M. Turan, C. P. Clementson, M. Sitti, ACS Appl. Mater. Interfaces 2016, 8, 5618.

[16] C. S. Boland, U. Khan, C. Backes, A. O'Neill, J. McCauley, S. Duane, R. Shanker, Y. Liu, I. Jurewicz, A. B. Dalton, J. N. Coleman, ACS Nano 2014, 8, 8819 .

[17] Y. C. Cai, J. Shen, Z. Y. Dai, X. X. Zang, Q. C. Dong, G. F. Guan, L. J. Li, W. Huang, X. C. Dong, Adv. Mater. 2017, 29, 1606411.

[18] F. L. Chen, Y. S. Gu, S. Y. Cao, Y. Li, F. Li, X. K. Zhang, M. X. Xu, Y. Zhang, J. Mater. Chem. C 2017, 5, 10571.

[19] C. H. Deng, L. J. Pan, D. M. Zhang, C. W. Li, H. Nasir, Nanoscale 2017, 9, 16404.

[20] F. Han, J. H. Li, S. F. Zhao, Y. Zhang, W. P. Huang, G. P. Zhang, R. Sun, C. P. Wong, J. Mater. Chem. C 2017, 5, 10167.

[21] Y. R. Jeong, H. Park, S. W. Jin, S. Y. Hong, S. S. Lee, J. S. Ha, Adv. Funct. Mater. 2015, 25, 4228.

[22] C. Lee, L. Jug, E. Meng, Appl. Phys. Lett. 2013, 102, 183511.

[23] J. T. Muth, D. M. Vogt, R. L. Truby, Y. Menguc, D. B. Kolesky, R. J. Wood, J. A. Lewis, Adv. Mater. 2014, 26, 6307.

[24] J. S. Noh, Nanoscale Res. Lett. 2013, 8, 441.

[25] Y. C. Tang, Z. B. Zhao, H. Hu, Y. Liu, X. Z. Wang, S. K. Zhou, J. S. Qiu, ACS Appl. Mater. Interfaces 2015, 7, 27432.

[26] A. P. Tjahyono, K. C. Aw, J. Travas-Sejdic, Sens. Actuators, B 2012, 166,426

[27] C. Y. Wang, X. Li, E. L. Gao, M. Q. Jian, K. L. Xia, Q. Wang, Z. P. Xu, T. L. Ren, Y. Y. Zhang, Adv. Mater. 2016, 28, 6640.

[28] C. Y. Wang, K. L. Xia, M. C. Zhang, M. Q. Jian, Y. Y. Zhang, ACS Appl. Mater. Interfaces 2017, 9, 39484.

[29] Y. Y. Xin, J. Zhou, X. Z. Xu, G. Lubineau, Nanoscale 2017, 9, 10897.

[30] C. Y. Yan, J. X. Wang, W. B. Kang, M. Q. Cui, X. Wang, C. Y. Foo, K. J. Chee, P. S. Lee, Adv. Mater. 2014, 26, 2022.

[31] T. T. Yang, W. Wang, H. Z. Zhang, X. M. Li, J. D. Shi, Y. J. He, Q. S. Zheng, Z. H. Li, H. W. Zhu, ACS Nano 2015, 9, 10867.

[32] J. C. Yeo, H. K. Yap, W. Xi, Z. P. Wang, C. H. Yeow, C. T. Lim, Adv. Mater. Technol 2016, 1, 1600018.

[33] M. C. Zhang, C. Y. Wang, H. M. Wang, M. Q. Jian, X. Y. Hao, Y. Y. Zhang, Adv. Funct. Mater. 2017, 27, 1604795.

[34] J. Zhou, H. Yu, X. Z. Xu, F. Han, G. Lubineau, ACS Appl. Mater. Interfaces 2017, 9, 4835.

[35] S. S. Duan, Z. H. Wang, L. Zhang, J. Liu, C. Z. Li, Adv. Mater. Technol. 2018, 3, 1800020.

[36] J. Y. Huang, D. W. Li, M. Zhao, A. R. Mensah, P. F. Lv, X. J. Tian, F. L. Huang, H. Z. Ke, Q. F. Wei, Adv. Electron. Mater. 2019, 5, 1900241.

[37] X. P. Jiang, Z. L. Ren, Y. F. Fu, Y. F. Liu, R. Zou, G. P. Ji, H. M. Ning, Y. Q. Li, J. Wen, H. J. Qi, C. H. Xu, S. Y. Fu, J. H. Qiu, N. Hu, ACS Appl. Mater. Interfaces 2019, 11, 37051. 
[38] G. J. Li, K. Dai, M. N. Ren, Y. Wang, G. Q. Zheng, C. T. Liu, C. Y. Shen, J. Mater. Chem. C 2018, 6, 6575.

[39] X. T. Li, H. B. Hu, T. Hua, B. G. Xu, S. X. Jiang, Nano Res. 2018, 11, 5799.

[40] B. H. Liang, Z. Zhang, W. J. Chen, D. W. Lu, L. L. Yang, R. L. Yang, H. Zhu, Z. K. Tang, X. C. Gui, Nano-Micro Lett. 2019, 11, 92.

[41] F. Pan, S. M. Chen, Y. H. Li, Z. C. Tao, J. L. Ye, K. Ni, H. Yu, B. Xiang, Y. B. Ren, F. X. Qin, S. H. Yu, Y. W. Zhu, Adv. Funct. Mater. 2018, 28, 1803221.

[42] H. Souri, D. Bhattacharyya, J. Mater. Chem. C 2018, 6, 10524.

[43] H. L. Sun, K. Dai, W. Zhai, Y. J. Zhou, J. W. Li, G. Q. Zheng, B. Li, C. T. Liu, C. Y. Shen, ACS Appl. Mater. Interfaces 2019, 11, 36052.

[44] Z. H. Tang, S. H. Jia, X. S. Shi, B. Li, C. H. Zhou, Polymers 2019, 11, 666.
[45] B. Tian, Q. Liu, C. S. Luo, Y. Feng, W. Wu, Adv. Electron. Mater. 2019, 6, 1900922

[46] Y. C. Wang, L. F. Zhu, D. Q. Mei, W. D. Zhu, J. Mater. Chem. C 2019, 7,8669

[47] M. X. Xu, F. Li, Z. Y. Zhang, T. Shen, J. J. Qi, Adv. Electron. Mater. 2019, 5, 1800461.

[48] Y. F. Yang, L. Q. Tao, Y. Pang, H. Tian, Z. Y. Ju, X. M. Wu, Y. Yang, T. L. Ren, Nanoscale 2018, 10, 11524.

[49] Y. J. Zhou, P. F. Zhan, M. N. Ren, G. Q. Zheng, K. Dai, L. W. Mi, C. T. Liu, C. Y. Shen, ACS Appl. Mater. Interfaces 2019, 11, 7405.

[50] D. J. Yonce, P. P. Bey, T. L. Fare, IEEE Trans. Circuits Syst. I-Fundam. Theor. Appl. 2000, 47, 273. 